\title{
Absolute frequency measurement of unstable lasers with optical frequency combs
}

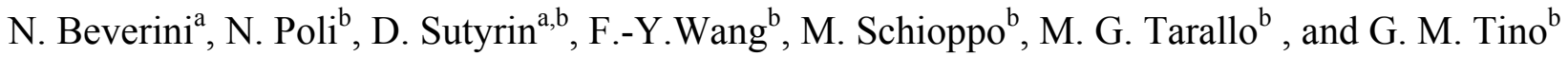 \\ ${ }^{a}$ Dipartimento di Fisica and CNISM, Università di Pisa; Largo Pontecorvo 3, Pisa ,Italy \\ ${ }^{\mathrm{b}}$ Dipartimento di Fisica e Astronomia, and LENS, Università di Firenze; Istituto Nazionale di Fisica \\ Nucleare, Sezione di Firenze, Polo Scientifico, 50019 Sesto Fiorentino, Italy
}

\begin{abstract}
Here we report on absolute frequency measurements of a commercial high power $\mathrm{CW}$ diode-pumped solid-state laser (Coherent Verdi-V5). This kind of lasers usually presents large frequency jitter (up to $50 \mathrm{MHz}$ ) both in the short term ( $1 \mathrm{~ms}$ time scale) and in the long term (>10 s time scale). A precise measurement of absolute frequency deviations in both temporal scales should require a set of different devices (optical cavities, optical wave-meters), each suited for measurements only at a specific integration time. Here we demonstrate how a frequency comb can be used to overcome this difficulty, allowing in a single step a full characterization of both short $(<1 \mathrm{~ms})$ and long term $\left(>10^{3} \mathrm{~s}\right)$ absolute frequency jitter with a resolution better than $1 \mathrm{MHz}$. We demonstrate in this way the flexibility of optical frequency combs for absolute frequency measurements not only of ultra-stable lasers but also of relatively unstable lasers. The absolute frequency calibration of the Verdi laser that we have obtained have been used in order to improve the accuracy of the measurements of the local gravitational acceleration value with ${ }^{88} \mathrm{Sr}$ atoms trapped in $1 \mathrm{D}$ vertical lattices.
\end{abstract}

Key words: femtosecond laser, frequency comb, absolute frequency measurement, Verdi, Dynamical laser instabilities, noisy laser behavior, cold atoms, gravity measurement.

\section{INTRODUCTION}

Today, optical frequency combs are widely used for many precision measurements, finding their natural application in frequency metrology for the calibration of ultrastable optical frequency references ${ }^{\mathrm{i}}$. Other interesting metrological applications of frequency combs has been described in the literature, in which less stringent requirements on frequency stabilization of the comb spectrum is required, as for example in calibrating astronomical spectra ${ }^{\text {ii }}$, or in molecular THz spectroscopy iii.

In this work, we report the implementation of a Ti:Sa femtosecond frequency comb for the characterization of the frequency stability of a commercial single-mode high power frequency doubled Nd-YVO laser (Verdi-V5 Coherent). This laser has found a wide application as a low noise pump for solid-state Ti:Sa lasers and is often employed in atomic physics experiments as a high power source for dipole trap. This laser is generally characterized by a low AM noise, but it can show a quite large frequency jitter $(>50 \mathrm{MHz})$ both in short term $(<1 \mathrm{~ms})$ and in long term $(>10 \mathrm{~s})$.

Here we demonstrate how an optical frequency comb can be used to measure the absolute frequency jitter at different temporal scales, showing how these device can find application also to perform precise absolute frequency characterization of unstable lasers. These measurements are of particular interest in the field of atomic physics in which high power CW solid state laser are typically employed to trap cold atoms in far off-resonance optical dipole traps with different geometries, ranging from single beam trap, crossed beam traps or multidimensional optical lattices. Especially in the last case, information about frequency jitter is important, since in this kind of trap laser frequency noise may convert in position fluctuations of the trap wells, resulting in additional atom losses.

Here the absolute calibration of Verdi laser emission frequency have been used to improve the accuracy of the measurements of the local gravitational acceleration value by the use of ${ }^{88} \mathrm{Sr}$ ultra-cold atoms trapped in 1D vertical realized with radiation from Verdi laser.

ICONO 2010: International Conference on Coherent and Nonlinear Optics, edited by Claude Fabre,

Victor Zadkov, Konstantin Drabovich, Proc. of SPIE Vol. 7993, 79931I · (c) 2011 SPIE

CCC code: $0277-786 \mathrm{X} / 11 / \$ 18 \cdot$ doi: $10.1117 / 12.880974$ 


\section{EXPERIMENTAL SETUP}

The experimental setup is described in Fig. 1. More details about the experimental setup can be found also in Ref. iv, v, vi, vii, viii. In brief, an ultracold sample of ${ }^{88} \mathrm{Sr}$ atoms in produced in a two-steps magneto-optical-traps operating on the dipole allowed ${ }^{1} \mathrm{~S}_{0^{-}}{ }^{1} \mathrm{P}_{1}$ transition at $461 \mathrm{~nm}$ and on the ${ }^{1} \mathrm{~S}_{0^{-}}{ }^{3} \mathrm{P}_{1}$ intercombination transition at $689 \mathrm{~nm}$. Atoms are then transferred in ${ }^{1} \mathrm{D}$ vertical lattice realized with about $2 \mathrm{~W}$ of laser radiation at $532 \mathrm{~nm}$ from a Verdi-V $5^{\circledR}$ laser. This system is employed for precision measurement of gravity through the observation of Bloch oscillation of trapped atoms ${ }^{6}$. The beam is vertically aligned and retro-reflected by a mirror, producing a standing wave with a period $\lambda_{L} / 2=266 \mathrm{~nm}$. In this condition, the acceleration of gravity $g$ is proportional both to the Verdi laser frequency and Bloch oscillation frequency through the formula $g=2 h v_{b} /\left(m_{S r} \lambda_{L}\right)$, where $m_{S r}$ is the atomic mass, $\lambda_{L}$ is the lattice wavelength, $v_{b}$ is the observed Bloch oscillation frequency, and $h$ is the Planck constant. Since $m_{S r}$ and $h$ are well known (relative uncertainty $5 \times 10^{-8}$ ), the force acting along the lattice axis can be determined by measuring the Bloch frequency $v_{b}$ and the wavelength of the light $\lambda_{L}$ emitted by the Verdi laser. To obtain absolute frequency measurements of Verdi, part of its radiation is then sent through a $200 \mathrm{~m}$ single-mode fiber to the laboratory where is operating the femtosecond frequency comb (see Fig.1).

Our optical frequency comb is based on an home-build femtosecond Ti:Sa laser with a pulse duration $50 \mathrm{fs}{ }^{4}$. The self-mode-locked laser has a repetition rate of about $294 \mathrm{MHz}$ and an average output power of more than $700 \mathrm{~mW}$ (in mode-locked operation) with $4.5 \mathrm{~W}$ pump power coming from solid-state Nd:Vanadate laser at $532 \mathrm{~nm}$. Following the availability, we have used for this purpose both a multi-mode laser (Spectra-Physics Millennia Xs ${ }^{\circledR}$ ) and a single-

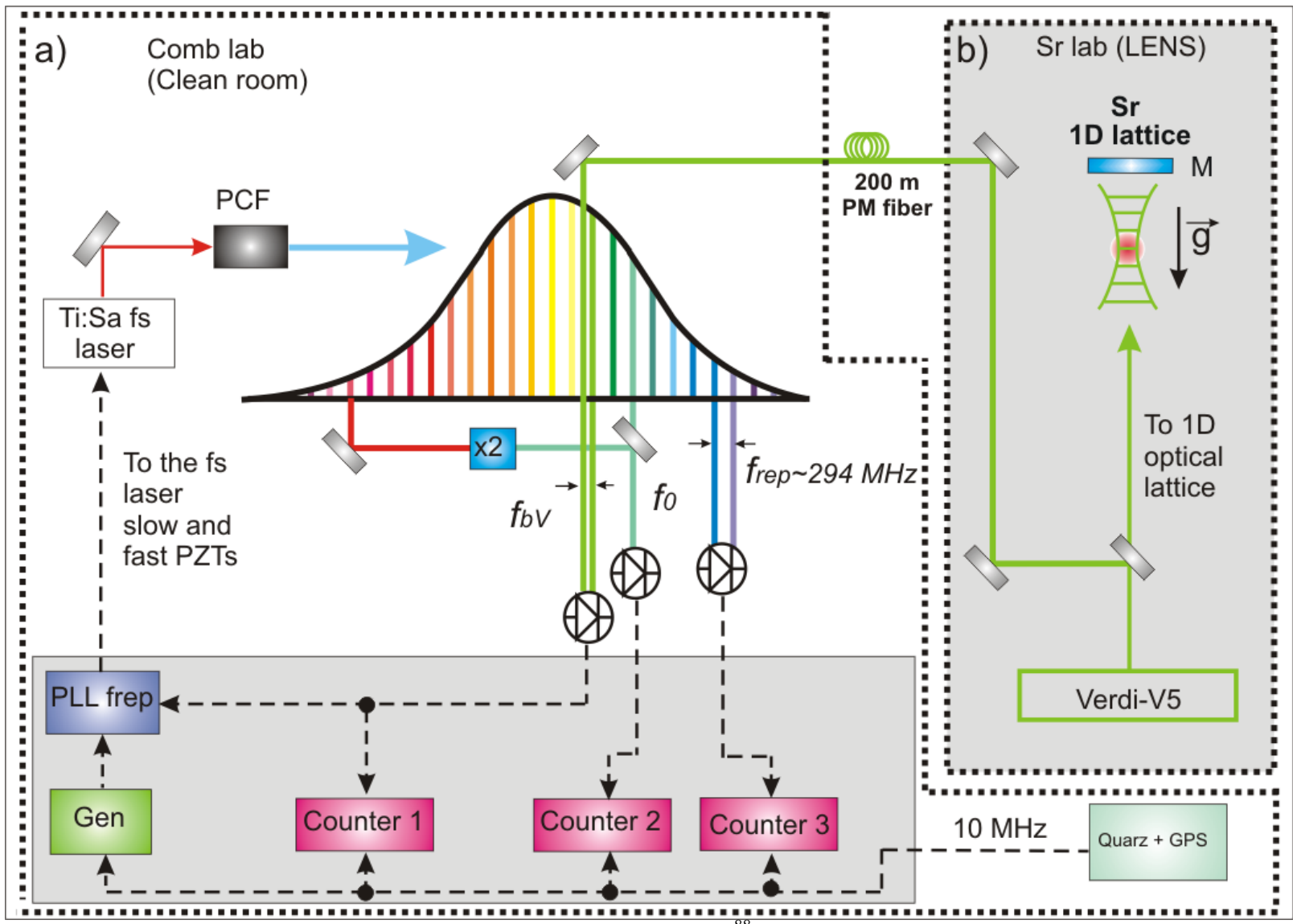

Figure 1: Experimental setup for Verdi frequency calibration and 1D ${ }^{88} \mathrm{Sr}$ optical lattice. The output radiation from Verdi is focused on the ultra-cold ${ }^{88} \mathrm{Sr}$ cloud (b) and part of it is then sent through $200 \mathrm{~m}$ fiber to the comb lab for frequency measurements (a). The beat-note signal between Verdi laser and the optical comb is used to stabilize the repetition rate of the femtosecond laser though PLL electronics that actuates slow and fast PZTs in the Ti:Sa cavity. All other interesting signals are 


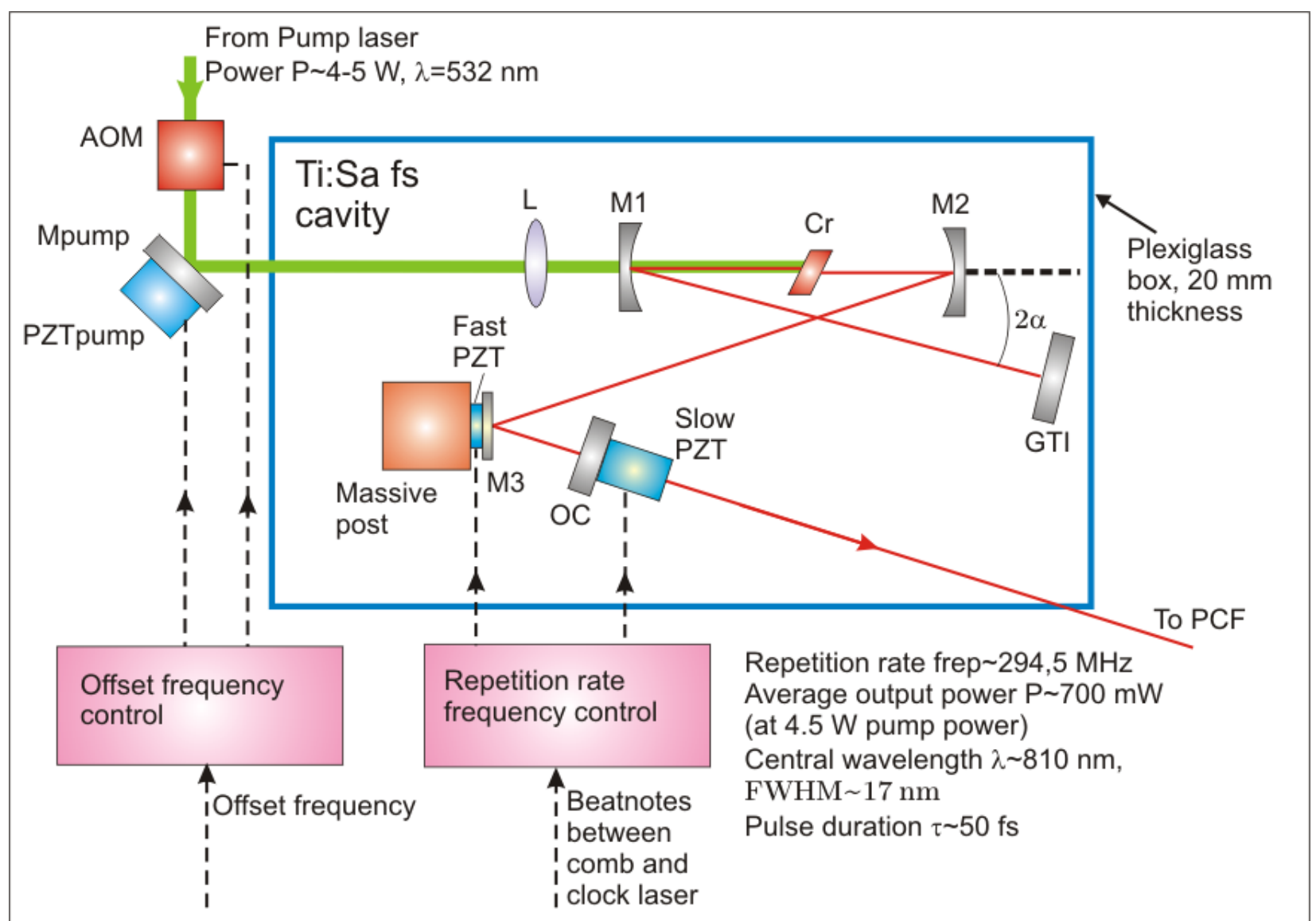

Figure 2: Experimental setup of Ti:Sa optical frequency comb cavity. L - lens, M1 and M2- curved mirrors, M3 - flat mirror, OC output coupler, GTI - Gires-Tournois interferometer mirror, $\alpha$ - astigmatism compensation angle, PCF - photonic crystal fiber.

mode laser (Coherent Verdi-V5 $\left.{ }^{\circledR}\right)$.

Fig.2 shows the design of the femtosecond laser with a detailed scheme of the Ti:Sa cavity. The fs laser cavity is composed by two curved mirrors (M1 and M2), one $2.5 \mathrm{~mm}$-long Ti:sapphire crystal (doped at $0.25 \%$ ) one high reflective Gires-Tournois interferometer type flat mirror (GTI) with dispersion $-550 \mathrm{fs}^{2}$, which is used to compensate the intracavity dispersion, and one output coupler (OC) with $3 \%$ transmission coefficient at $800 \mathrm{~nm}$. An additional 1/4" inch diameter flat folding mirror (M3) with zero dispersion has been inserted in the cavity to allow fast (up to $40 \mathrm{kHz}$ ) control of the cavity length through a PZT. The overall estimated intracavity dispersion is about $-200 \mathrm{fs}^{2}$, while the total length of the cavity is $\sim 510 \mathrm{~cm}$.

All the mirrors (except the output coupler) are dielectric with $>99 \%$ reflection coefficient at the center wavelength of $800 \mathrm{~nm}$. The curved mirrors (radius of curvatures $25 \mathrm{~cm}$ ) are both aligned to form X-shape cavity with an incidence angle $\alpha=8^{\circ}$ that compensates the crystal astigmatism. The pump beam is coupled into the cavity through the curved mirror M1 and is focused on the crystal through a lens with focal length $7.5 \mathrm{~cm}$. The flat side of the input mirror M1 is AR coated at $532 \mathrm{~nm}$ to minimize losses. A second piezoelectric transducer on the OC mirror of the Ti:Sa cavity allows the slow control of the cavity length.

The emission spectrum of the Ti:Sa mode-locked laser is centered around $810 \mathrm{~nm}$ with a typical FWHM of about $17 \mathrm{~nm}$. The laser output is then coupled into a photonic crystal fiber (Femtowhite $800^{\circledR}$ ) that broadens the spectrum up to a full optical octave (from $400 \mathrm{~nm}$ up to $1100 \mathrm{~nm}$ ). The maximum power obtained at the fiber output is $350 \mathrm{~mW}$, but in order to reduce the wideband frequency noise generated in the fiber at high intensity, we usually work with less than $250 \mathrm{~mW}$. 
The carrier envelope offset (CEO) frequency $f_{C E O}$ is measured with a standard $f-2 f$ interferometer ${ }^{\text {ix }}$ with a nonlinear 3mm-long BBO crystal (in type I configuration) placed in the first interferometer arm. The slow drift of offset frequency is corrected by actuating through a PZT on the position of the pump mirror (PM), while the fast fluctuations (up to $100 \mathrm{kHz}$ ) are corrected by acting on pump power through the use of an acusto-optical modulator (AOM).

The common way to stabilized the comb repetition rate is either to lock it to an RF standard or, to increase its stability, to lock the beatnote between one tooth of the frequency comb and an optical standard. This is done typically by using PLL servo electronics acting on cavity PZTs. Note that, in the case of direct stabilization in the optical domain, the frequency stability of the optical standard will transfer to all comb components, and the frequency noise on the repetition rate signal is reduced by a factor $n$ :

$$
f_{\text {rep }}=\left(f_{V} \pm f_{C E O} \pm f_{b}\right) / n,
$$

where $f_{V}$ is the Verdi frequency, $f_{C E O}$ is the CEO frequency, $f_{b}$ is the beat-note between a comb tooth and the optical standard and $n$ is an integer defining the comb tooth that has a value of the order of $10^{6}$.

\section{EXPERIMENTAL RESULTS}

In our case, we want to measure a laser frequency characterized by a quite large jitter. The output radiation from the 200-m fiber transfer is superimposed in a polarization beam-splitter with the radiation from the femtosecond comb (filtered at $\lambda=532 \pm 5 \mathrm{~nm}$ ). The beat signal between the Verdi laser and the nearby tooth of the comb Is detected on a fast photodetector and observed on a rf spectrum analyzer. A typical registration is presented in Fig.3a, which evidences that in a time scale of a few ms, the linewidth is about $10-15 \mathrm{MHz}$ broad, due to Verdi laser fast frequency jitter ms ${ }^{\mathrm{x}}$. Due to the typical low signal to noise observed ( $<25 \mathrm{dBm}$ on a $300 \mathrm{kHz}$ bandwidth) and the fast frequency jitter, it is not possible to use standard frequency counters to perform precise frequency measurements.

We overcome this difficulty by locking the repetition rate of the comb to this unstable reference, stabilizing the beatnote frequency.

For this purpose, after the detection on the photodiode, the beat note signal is amplified, filtered and split in two channels. One channel is used to monitor the comb stabilization, while the second one is sent to a phase-frequency detector (PFD). The reference signal for the stabilization (sent to the second PFD input) is given by a local $30 \mathrm{MHz}$ generator, referenced at long term to GPS reference signal. The servo signal for stabilization generated by the PFD (that is proportional to phase difference between the two input signals) is then sent to a PID (proportional-integratedderivative) controller that is used to actuate two PZT attached to two comb cavity mirrors for slow and fast (up to 40 $\mathrm{kHz}$ ) cavity length change.
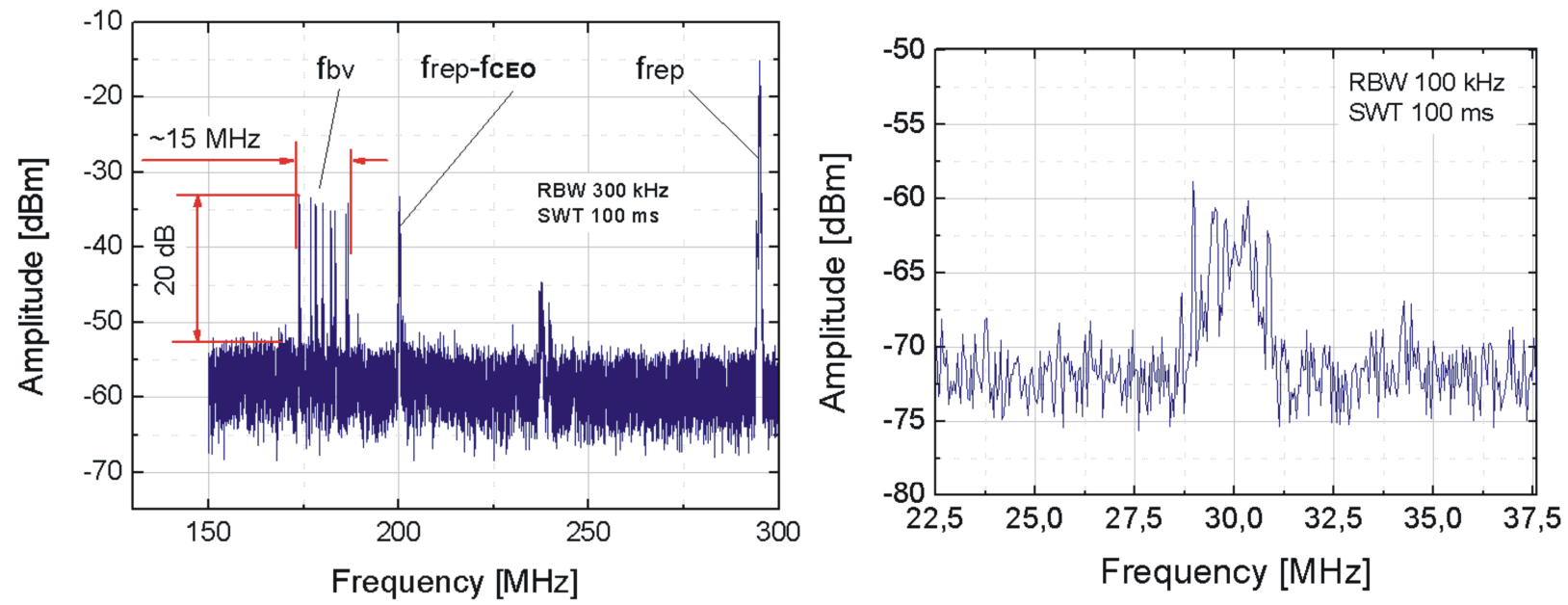

Figure 3: Beat-notes between Verdi and the optical comb, a) left, free-running regime, b) right, with the comb repetition rate locked on the beat-note signal. 
After detection the beat note signal is amplified, filtered and split in two channels. One channel from RF power splitter is used to monitor the comb stabilization, while the second one is sent to a phase-frequency detector (PFD).The reference signal for the stabilization (sent to the second PFD input) comes from a local $30 \mathrm{MHz}$ generator, referenced at long term to GPS reference signal. The servo signal for stabilization generated by the PFD (that is proportional to phase difference between the two input signals) is then sent to a PID (proportional-integrated-derivative) controller that is used to actuate two PZT attached to two comb cavity mirrors for slow and fast (up to $40 \mathrm{kHz}$ ) cavity length change.

In lock condition, as shown in (Fig. 3), the beat-note signal is stabilized at $30 \mathrm{MHz}$ and the frequency jitter is then imposed on frequency comb repetition rate. Thanks to the large de-multiplication factor of the comb $\left(\sim 1.9 \times 10^{6}\right)$ the $10-15 \mathrm{MHz}$ frequency jitter at $532 \mathrm{~nm}$ gets divided down to about $1 \mathrm{~Hz}$ and imposed on the repetition rate signal that is typically observed with $\mathrm{S} / \mathrm{N}>50 \mathrm{~dB}$ over a bandwidth of $300 \mathrm{kHz}$. The repetition rate signal is then easily counted with a frequency counter set with $1 \mathrm{~s}$ gate time.

In order to determine the absolute frequency of the Verdi we also count the CEO frequency. In our setup this signal is sufficiently long term stable compared to Verdi frequency instabilities, so that a stabilization of this signal was not required for this measurement. In this case, the absolute value of the Verdi frequency is given by equation $f=n \cdot f_{\text {rep }} \pm f_{C E O} \pm f_{b}$. To determine the correct tooth number at the beginning and at the end of each measurement we employ a lambda meter ${ }_{2}$ calibrated with a stabilized laser resonant with the $689 \mathrm{~nm}{ }^{1} \mathrm{~S}_{0}{ }^{3} \mathrm{P}_{1}{ }^{88} \mathrm{Sr}$ transition, that allows an evaluation of the Verdi laser frequency with an uncertainty of the order of $100 \mathrm{MHz}$.

In Fig. 4 the result of an absolute frequency measurement that lasts for about $10^{4} \mathrm{~s}$ is shown. Long term deviation of Verdi frequency greatly depends on many factors, mainly related to temperature, and laser power setting. To increase long term stability we performed this measurement after that the laser was operating continuously for a long time (about two days) at the same power setting. Moreover, we covered the laser head with thermal insulation foam to increase the passive temperature stability

From this measurement we can clearly see periodical deviation of the frequency at two different regimes. Firsts, we observe a long-term oscillation of the Verdi frequency with period $\sim 1000 \mathrm{~s}$ and amplitude $130-140 \mathrm{MHz}$. Secondly, there is a fast oscillation with a shorter period $\sim 33 \mathrm{~s}$ and an amplitude $30-70 \mathrm{MHz}$ with typical value $30-50 \mathrm{MHz}$ (see Fig. 4 inset). The missing data in this set are caused to a comb unlocking from the Verdi signal.

We think that this represents a lower limit for long-term frequency fluctuations of non-stabilized Verdi laser for the reasons explained above. After shutdown period, these lasers can show in fact both quite different absolute frequency (offset by several $\mathrm{GHz}$ ) and large frequency drift rate at start up (up to $100 \mathrm{MHz}$ in one minute).

As shown in the inset of Fig. 4, absolute determinations of the instantaneous value of the Verdi frequency (in the case of the measurement shown in Fig. 4 the mean value is $563257825 \pm 75 \mathrm{MHz}$ ) can be in this way performed with a sub-MHz resolution. This value can eventually be used to determine gravity with $10^{-7}$ relative uncertainty, by observing at the same time Bloch oscillation of ultra-cold ${ }^{88} \mathrm{Sr}$ atoms trapped in vertical standing wave.

\section{CONCLUSION}

We made the absolute frequency measurement of the Verdi-V5 with sub-MHz resolution and observed the fast and the slow behavior of its frequency by the use of a femtosecond frequency comb. The frequency measurement have been used in accurate determination of gravity by the use of ${ }^{88} \mathrm{Sr}$ atoms trapped in vertical lattices. This work demonstrates the great flexibility of optical frequency combs for precision measurements of optical frequencies of unstable lasers. This study in turn may also be important for the study of stabilization technique of CW solid-state diode pumped lasers and moreover could be important for the study of Ti:Sa femtosecond lasers dynamics.

We want acknowledge the group of the Institute of Laser of the Russian Academy of Science in Novosibirsk for the help in the developing our comb frequency system. In particular, we thanks dr. Sergey Chepurov, whose expertise was fundamental for making operative the comb system. One of the authors (F.-Y. Wang) undertook this work with the support of "ICTP Program for Training and Research in Italian Laboratories, Trieste, Italy" 


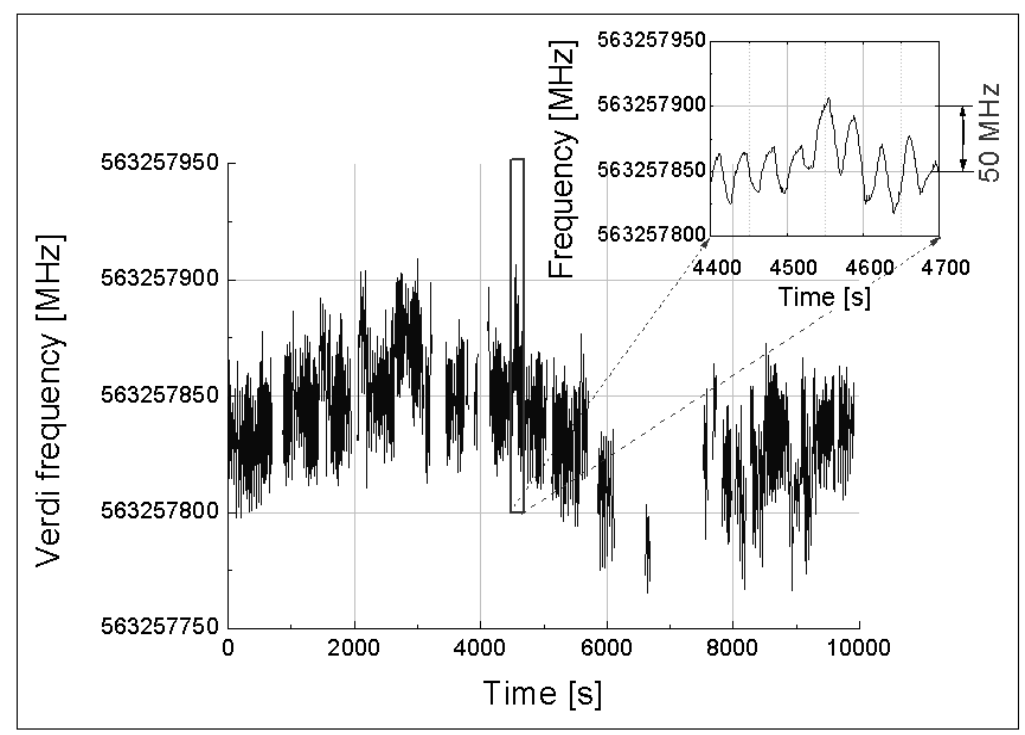

Figure 4: Verdi Absolute frequency measurement. In the inset are shown the Verdi frequency fluctuations at shorter time scale.

\section{REFERENCES}

[i] T. Udem, R. Holzwarth, and T. W. Hänsch, "Optical Frequency Metrology", Nature 416, 233-237 (2002)

[ii] T. Steinmetz et al., Laser Frequency Combs for Astronomical Observations, Science 321, 1335-1337 (2008)

[iii] N. Beverini, G. Carelli, A. De Michele, E. Maccioni, B. Nyushkov, F. Sorrentino, A. Moretti: Coherent multiwave heterodyne frequency measurement of a FIR laser by means of a femtosecond laser comb, Opt. Lett. 30, 32-34 (2005) [iv] N. Poli, M. G. Tarallo, M. Schioppo, C. W Oates, S. Chepurov, D.Sutyrin, A. De Michele, N. Beverini and G. M. Tino, "Strontium Optical Lattice clock" Proc. of the 5th International Symposium "Modern Problems of Laser Physics" (MPLP'2008) Novosibirsk, Russia, August 2008, pp. 327-334 (2008).

[v] N. Poli, M. G. Tarallo, M. Schioppo, C. W. Oates and G. M. Tino, "A simplified optical lattice clock", Appl.Phys. B 97, 27-33 (2009)

[vi] N. Poli, G. Ferrari, M. Prevedelli, F. Sorrentino, R.E. Drullinger, G.M. Tino, "Laser sources for precision spectroscopy on atomic strontium", Spectrochimica Acta A 63, 981-986 (2006)

[vii] F. Sorrentino, G. Ferrari, N. Poli, R. E. Drullinger, G. M. Tino, "Laser cooling and trapping of atomic strontium for ultracold atoms physics, high-precision spectroscopy and quantum sensors", Mod. Phys. Lett. B 21, 1287-1320 (2006)

[viii] G. Ferrari, N. Poli, F. Sorrentino, G. M. Tino, "Long-Lived Bloch Oscillations with Bosonic Sr Atoms and Application to Gravity Measurement at the Micrometer Scale”, Phys. Rev. Lett., 97, 60402 (2006)

[ix] H. R. Telle, G. Steinmeyer, A. E. Dunlop, J. Stenger, D. H. Sutter, U. Keller, "Carrier-envelope offset phase control: A novel concept for absolute optical frequency measurement and ultrashort pulse generation", Appl. Phys. B 69, 327 (1999)

[x] Coherent report: "Wavelength Control White Paper". Web link: www.coherent.com/Lasers/index.cfm? fuseaction=show.page\&id=1852 